\title{
Automated Bucket Stabilizer for Hydraulic Mobile Machine
}

\author{
Jarno R. A. Uusisalo, Jani M. Vilenius, Tomi R. Krogerus, Otso I. Karhu, and Kalevi J. Huhtala
}

\begin{abstract}
A small general-purpose teleoperated hydraulic mobile machine is studied at the Institute of Hydraulics and Automation at the Tampere University of Technology. The boom and the bucket of the machine are normally controlled with a joystick. Horizontal movement of the joystick controls the bucket and vertical movement controls the boom. An angle of the bucket, compared to the horizontal level changes while the boom is moved up and down, unless the change of a boom angle is compensated by controlling the bucket manually at the same time. However, compensation is difficult during the driving. This paper describes a bucket stabilizer system which compensates the change of the boom angle automatically and makes so controlling of the machine easier for an operator.

A mechanical structure of the machine causes some limitations for the bucket stabilizer system. For instance, the angle of the bucket can not be maintained while the boom is moved if the bucket is already in the extreme position. These special situations are considered and error conditions are prevented.

The angle of the boom and the bucket compared to the horizontal level are measured with help of accelerometer sensors. The calculation operations are distributed. On this account, the system is independent from existing control electronics of the machine. Communication between the control electronics of the machine and the bucket stabilizer system is carried out with CAN bus. The structure of the electronics of the system is introduced in this paper. Also the design and implementation of the control algorithm are introduced. The measured results of the automated bucket stabilizer system are shown. Simulated results are compared to measured values. User experiences are also enclosed.
\end{abstract}

Index Terms-CAN, hydraulic, proportional valve, stabilizer.

J. R. A. Uusisalo is with the Institute of Hydraulics and Automation, Tampere University of Technology, Finland (corresponding author to provide phone: $\quad+358-3-3115-4485 ; \quad$ fax: $+358-3-3115-2240$; $\quad$ e-mail: jarno.uusisalo@tut.fi).

J. M. Vilenius is with the Institute of Hydraulics and Automation, Tampere University of Technology, Finland (e-mail: jani.m.vilenius@tut.fi).

T. R. Krogerus is with the Institute of Hydraulics and Automation, Tampere University of Technology, Finland (e-mail: tomi.krogerus@tut.fi).

O. I. Karhu is with the Institute of Hydraulics and Automation, Tampere University of Technology, Finland (e-mail: otso.karhu@tut.fi).

K. J. Huhtala is with the Institute of Hydraulics and Automation, Tampere University of Technology, Finland (e-mail: kalevi.huhtala@tut.fi).

\section{INTRODUCTION}

$\mathrm{H}$ YDRAULICALLY operated mobile machine are common nowadays. Typically there is some kind of boom in the machine and an attachment which is connected to the boom. In the studied machine the attachment can easily be changed. The attachment range is large [1]. For instance, many kind of buckets and pallet fork can be used in the same machine without any changes in the machine. In this paper, the attachment which is connected to the boom is generally called bucket. Also measurements have been made with general bucket [1]. However, it is good to remember that the stabilizing system can be used also with other attachments as well as with general bucket.

The boom of the machine is controlled by the vertical movement of the joystick and the bucket is controlled by the horizontal movement of the joystick. It is often desired to keep the angle of the bucket compared to horizontal level constant while moving boom up and down. However, keeping the angle constant manually by controlling the valve of the bucket while moving the boom is difficult. On this account, an automated stabilizer system is needed.

The electrical and mechanical structure of the automated bucket stabilizer system is introduced in this paper. The system uses existing hydraulic of the machine. Critical part of the hydraulics of the machine and an effect of the characteristics of the hydraulics for the stabilizer system are discussed. Also a control algorithm is introduced. Finally some measurements and user experiences are described.

\section{IMPLEMENTATION OF THE SYSTEM}

\section{A. Mechanical Structure}

The body of the original machine has not been changed. Only the electronics and the proportional valve block have been installed outside the body of the studied machine. The modified machine is shown in the Fig. 1. The position of the proportional valve block and the electronic components are marked in the Fig. 1 with numbers from one to five.

It deserves consideration that a centre of gravity of the machine is quite near to front tires. A mass of the boom and the bucket is significant compared to a mass of the machine. Caused by this, the whole machine is oscillating a bit while the boom is moved. 


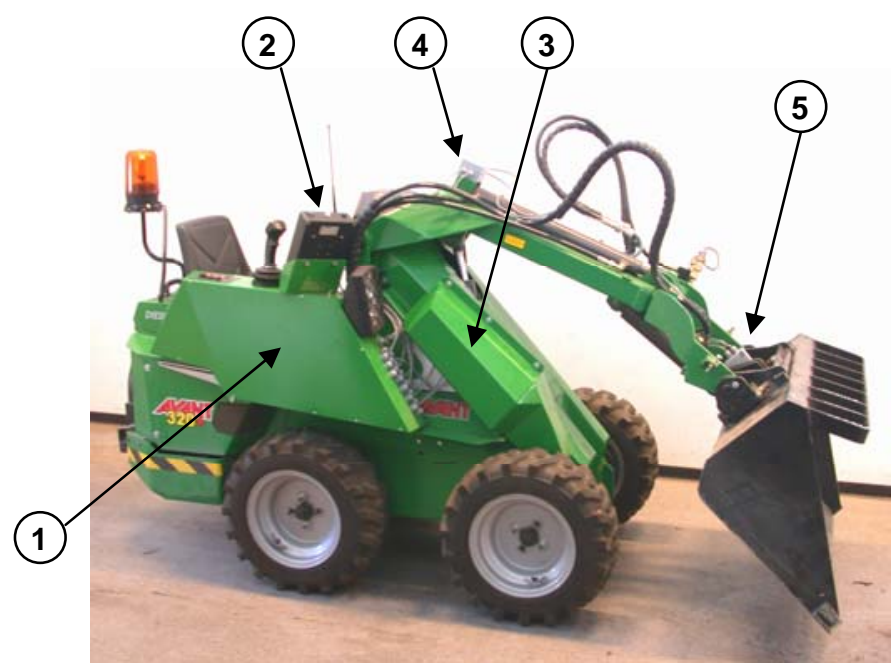

Fig. 1. The studied hydraulic mobile machine. Numbered items in the picture: (1) Electronic components, (2) Control and display module, (3) Proportional valve, (4) Controller module of the stabilizer system, (5) Bucket inclinometer. [3]

\section{B. Hydraulics}

On/off-valves are used to control both hydraulic power transmission and actuators of the original mobile machine. On/off-valves have been replaced with electrically controlled closed centre position proportional valve in the studied machine. Proportional valve is designed for mobile applications. The maximum flow per section is $40 \mathrm{l} / \mathrm{min}$ and the maximum pressure is 210 bar [2]. However, the maximum pressure of the system is limited to 175 bar. The used valve is relatively slow with maximum frequency about $5 \mathrm{~Hz}$. [3]

The proportional valve is not selected specially for stabilizer application. It is selected primarily to enable electrical proportional control for whole machine, including hydraulic power transmission. The physical size and pricequality ratio have been part of selection criteria. Even though the properties of the used proportional valve are not optimum for this application, another valve has not been wanted to use in stead of existing valve.

Both the boom and the bucket of the machine are moved by hydraulic cylinder. Size of boom cylinder is $63 / 32-360$ and size of the bucket cylinder is 63/32-150. There are about four meters hydraulic hoses both before and after the bucket cylinder. The diameter of the hoses is $10 \mathrm{~mm}$.

\section{Electronics}

When stabilizer system is not enabled, all control operations of the machine are carried out in the display module which is marked in the Fig. 1 with number 2. There are two IO-modules inside green box which is marked in the Fig. 1 with number 1 . The IO-modules are reading states of pressure sensors (LS pressure and supply pressure) and a joystick as an input and controlling the valve as an output. Communication between these modules is carried out with CAN bus. Proportional valve of the studied machine is controlled with pulse width modulated (PWM) signals. These
IO-modules generate pulse width modulated signals which are proportional to CAN message. [4]

While stabilizer system is enabled, only hydraulic power transmission is controlled by means of the display module. Control messages for the boom and the bucket are calculated in the controller module which is marked in the Fig. 1 with number 4. A digital signal processor DSP56F803 from Motorola is used to carry out controller of the system [5]. There is also an inclinometer SCA610-CA1H1G inside the box 4 [6]. The inclinometer is used to find out the angle of the boom compared to horizontal level.

An inclinometer, made by Axiomatic is used to measure the angle of the bucket. The inclinometer is marked in the Fig. 1 with number 5. The range of the used inclinometer is 360 degrees and the resolution is 0.05 degrees [7]. The inclinometer transmits the value of the angle to the controller module through CAN bus. $125 \mathrm{kbit} / \mathrm{s}$ baud rate is used for CAN bus.

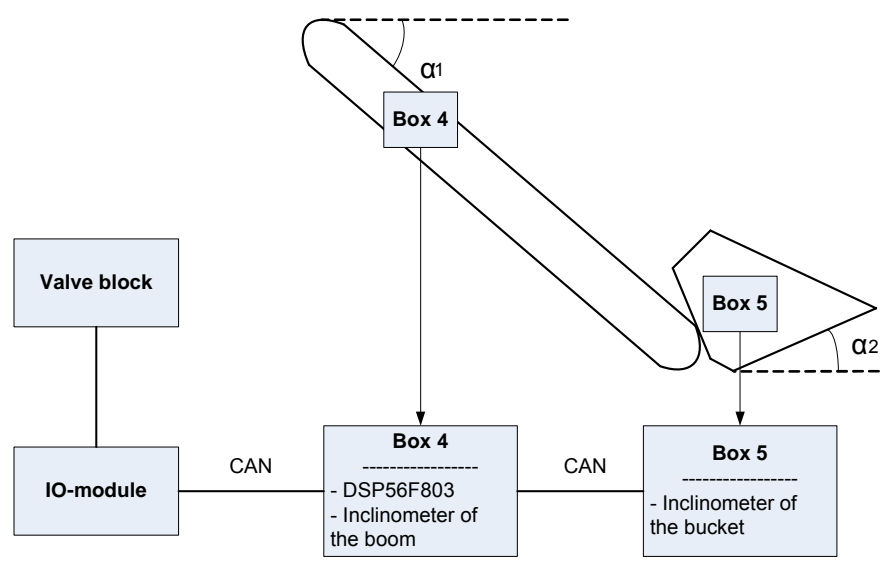

Fig. 2. A principle picture of the stabilizer system.

\section{CONTROL AlgORITHM}

The control algorithm of the stabilizer system is carried out with P-controller. P-controller is the most suitable solution for this application since the system is controlling a cylinder which already works as an integrator. With P-controller a sufficient accuracy for the application is achieved. Also PIcontroller was tested. However, P-controller behaved better.

As mentioned, the proportional valve is not optimum choice for this application. The valve is too slow for accuracy control with higher speeds. Very high accelerations of the boom cause delay for compensation of the bucket angle. For instance, if the boom descents very fast, an error of the angle of the bucket increases at first. The error approaches to zero when the boom stops. Raising the gain of the P-controller makes the system unstable. On this account, ramp functions for boom control have been carried out to prevent very high accelerations.

Another disadvantage of the valve is that maximum flow through the valve is quite large. The error of the bucket angle is always zero when the stabilizer system is enabled. P- 
controller tends to keep error in zero. On this account, the error is always quite small and so also an opening of the valve is small. This makes accuracy control difficult, particularly when valve is controlled with PWM. A PWM output of the used IO-modules can be controlled with $0.1 \%$ resolution which means that there are 1000 available steps between zero volt and supply voltage. However, the operational area of the valve is from $25 \%$ to $75 \%$ of supply voltage, which limits the useful control area of the PWM output to 500 steps. Also a quite large dead zone of the valve limits the control area. Because of these reasons, when the error is small, let say below five degrees, there are only dozen different control signals available for the valve. On this account, very small error will not open the valve at all, which means that there will always exist small steady-state error in the system. However, limited accuracy or small steady-state error are not significant for application.

\section{A. Compensation of the Cylinder}

The cylinder of the bucket is asymmetric. On this account, behavior of the bucket depends on direction. This asymmetry is compensated with software by using different gain for both directions (up and down). Gain factors are proportional to ratio of piston area and piston rod area of the cylinder. The right ratio of gain factors were also found by testing the system.

\section{B. Compensation of the Valve Dead Zone}

There is quite large dead zone in the used proportional valve. Dead zone compensation is carried out with software. When there is an error in the actual angle of the bucket compared to set angle, the controller controls the valve so that spool moves right away on the dead zone edge. After that starts normal P-control. By skipping the dead zone, control becomes faster and at the same time flow-spool opening curve is almost linear on the whole control area.

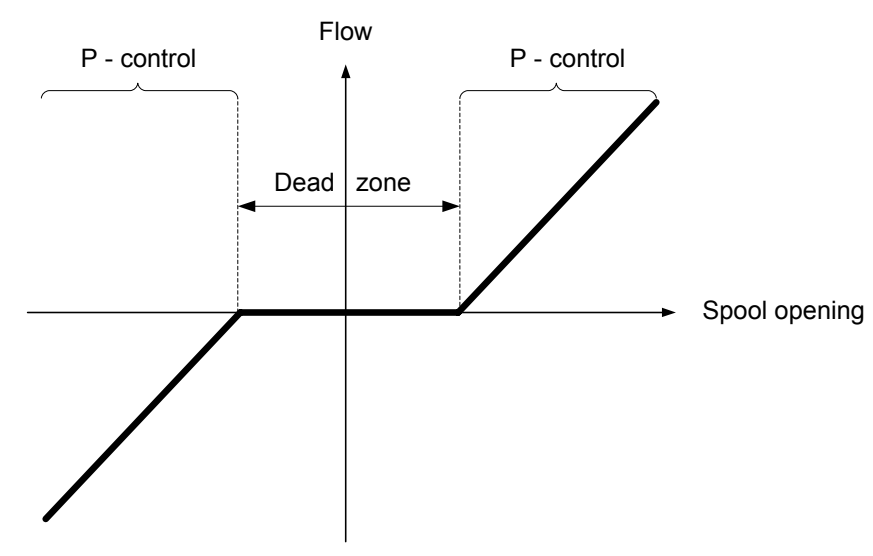

Fig. 3. Principled flow-spool opening curve of the proportional valve. Actual $\mathrm{P}$-control is used on two areas which are marked with brackets (P-control).

\section{Limiting THE MOVEMENT OF THE BOOM IN EXCEPTIONAL CASES}

The stabilizer system tends to keep the bucket in the same angle that it was when the system was enabled. The bucket is not necessarily at the horizontal level. The mechanical structure of the machine may make the control of the bucket impossible even though the boom could still move. Let us consider as an example a situation where the set value of the bucket angle is close to minimum (the bucket point to ground) and the boom is close to lowest position (Fig. 4, $\alpha_{2}$ ). When the boom starts to rise the bucket should be controlled downward to keep the angle in the set value. However, the angle between the boom and the bucket (angle $\alpha$ ) can not be lower than $\alpha_{1}$ or higher than $\alpha_{2}$ because of the mechanical structure of the machine. If these exceptional cases are not considered, a change in the angle of the boom can not be compensated and an error of the bucket angle will increase. It is very difficult for operator to see what is the highest position for the boom where the system is still stabilizing the bucket. On this account, these limitations have to be taken care by the stabilizer system.

There are all together two exceptional cases. One is when the angle between the boom and the bucket is minimum $\left(\alpha_{1}\right)$ and the other is when the angle is maximum $\left(\alpha_{2}\right)$. Detection and reacting of these two exceptional cases is carried out by measuring also the angle of the boom in addition to the angle of the bucket. If the angle between the boom and the bucket is going to be smaller than minimum or bigger than maximum the system controls the valve of the boom to the centre position and stops the move of the boom though an operator would still try to move the boom. So the system prevents automatically increasing error of the bucket angle caused by mechanical structure of the machine.

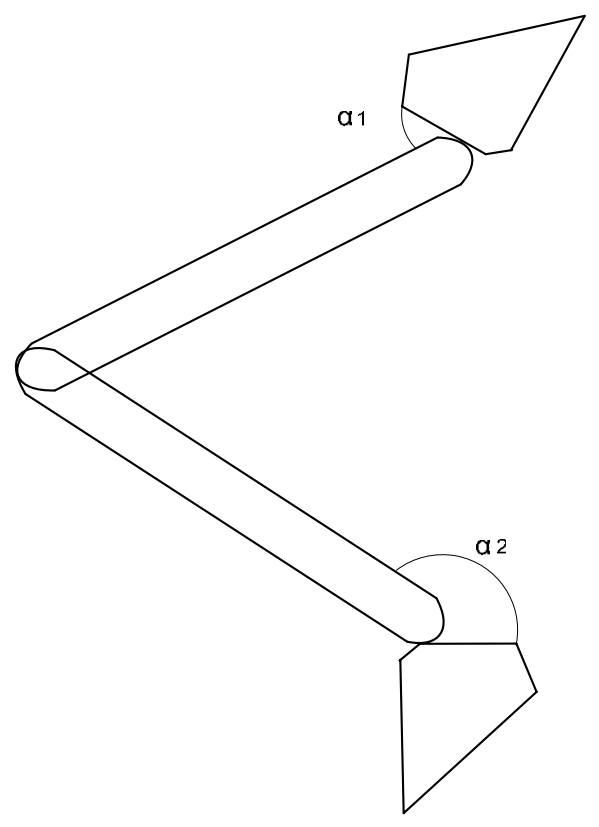

Fig. 4. A principle figure of the boom and the bucket in extreme positions. 


\section{RESUlts}

A behavior of the stabilizer system was tested in the laboratory of the Institute of Hydraulics and Automation. The boom of the machine was moved up and down by controlling the joystick which is a normal way to control the boom. The angles of the bucket and the boom were measured with dSpace. An extra inclinometer was fastened to the boom during the measurements to find out an accurate angle of the boom. The extra inclinometer was similar with the inclinometer of the bucket. These two inclinometers were connected to dSpace through CAN bus. The machine was in its place on even cement floor. Testing arrangement was tend to make correspond to normal working conditions. No load was used in the bucket.

Measurements were made with three different angular speed of the boom to both directions. At first, the boom was moved up and down with minimum speed. It means that opening of the valve was limited. Then same measurements were repeated with medium angular speed which can consider to be normal way to control the boom with load. Finally same measurements were made with maximum angular speed and without any ramp functions. Fig. 5-10 consist of two parts. A set angle is plotted with red color and an actual angle of the bucket is plotted with blue color in upper part of Fig. An angle of the boom during the motion is plotted with blue color in lower part of Fig. On the X-axis there is a time in seconds and on the $\mathrm{Y}$-axis an angle in degrees. All angles are in proportion to horizontal level.

Quite high peaks (from one to two degrees) in the angle of the bucket right after the boom has started to move and just before the stops moving can be discovered almost in all measurements. These peaks are caused by oscillation of the whole machine. This phenomenon can be discovered from the curve of the boom angle. There are peaks in to wrong direction in the angle of the boom when the angular speed changes rapidly. The proportional valve is so slow that stabilizer can not cut these peaks away. The only way to filter these peaks is to limit accelerations of the boom.

\section{A. Slow Angular Speed}

Angles during a slow motion of the boom upward are plotted in the Fig. 5. The angle of the boom changes about 70 degrees in 23 seconds. The set angle of the bucket is 92.1 degrees. An error in the angle of the bucket increases when boom starts to move. A tracking error sets to about 2.5 degrees which is good. After the motion of the boom is stopped the controller corrects the tracking error. A steadystate error is about 1.5 degrees. The steady-state error is caused by P-controller. Also the lack of accuracy in PWM control affect to steady-state error. However, this error is not disturbing for the application.
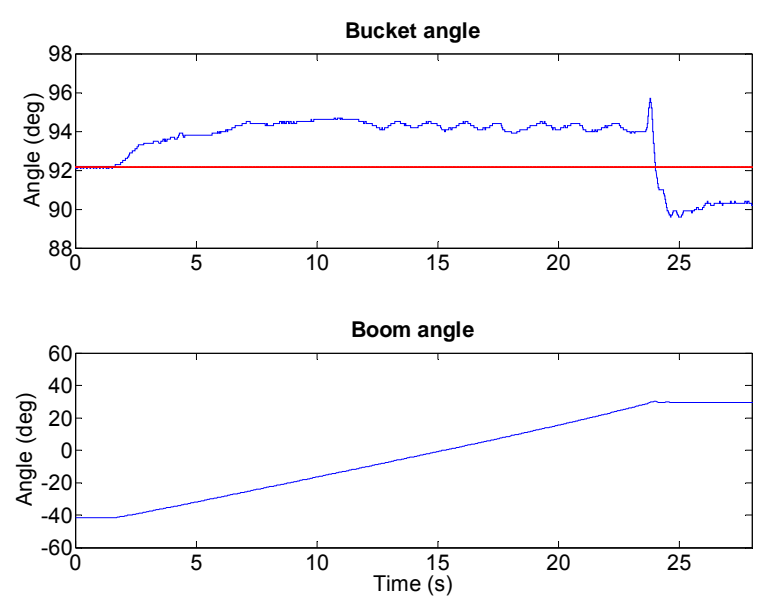

Fig. 5. Angles while the boom is moving slowly upward.

Similar measurement was made while boom was moved downward (Fig. 6). The angle of the boom changed about 60 degrees in 14 seconds. The angular speed is faster than it was when the boom moved upward. This is caused by gravity. The boom does not need as much force when it is coming down than it needs when it is going up.

The tracking error is now less than two degrees and the steady-state error is less than 0.5 degrees. These values are very good. Even the highest peaks, caused by oscillation of the machine are only about three degrees.
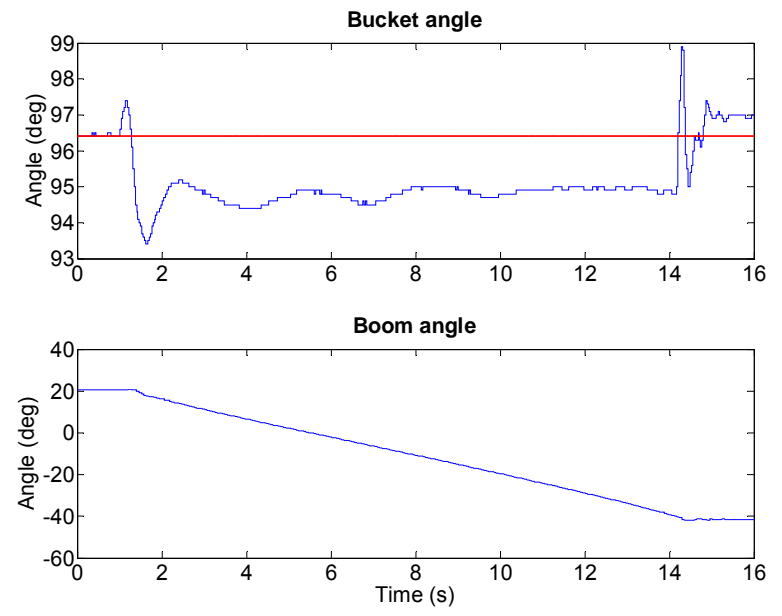

Fig. 6. Angles while the boom is moving slowly downward.

\section{B. Medium Angular Speed}

In the medium angular speed measurements the angle of the boom changed about 65 degrees in 10 seconds (Fig. 7). As it can be noticed from the Fig. 7 the tracking error is about 4.5 degrees and steady-state error is less than 0.5 degrees. 

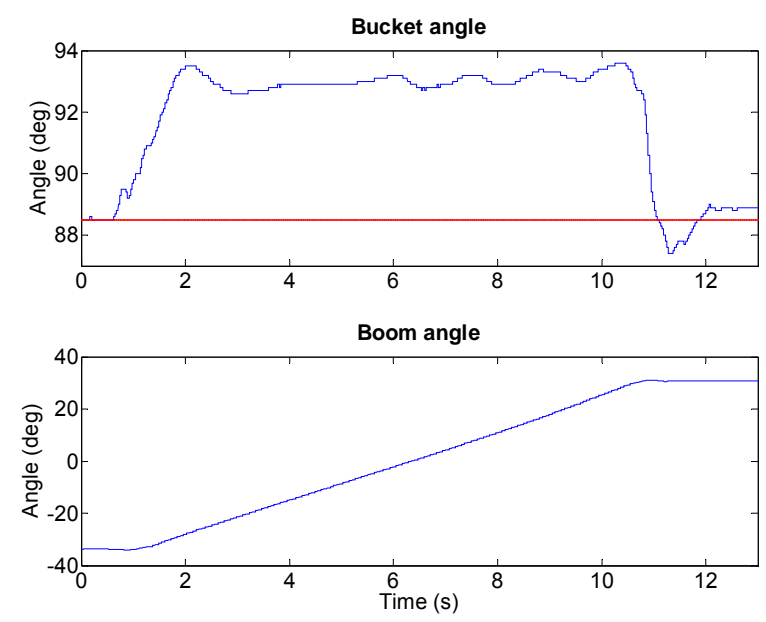

Fig. 7. Angles while the boom is moving upward with medium angular speed.

In the Fig. 8 the boom was moved downward with medium angular speed. The motion actually lasted approximately as long as it lasted with minimum angular speed. It is very difficult to limit the angular speed of the boom when it is moving downward. Even minimum opening of the valve moves the boom quite fast because of gravity.

The tracking error is approximately same that it was when the boom was moved with minimum speed. However, now the steady-state error is smaller, less than 0.5 degrees.
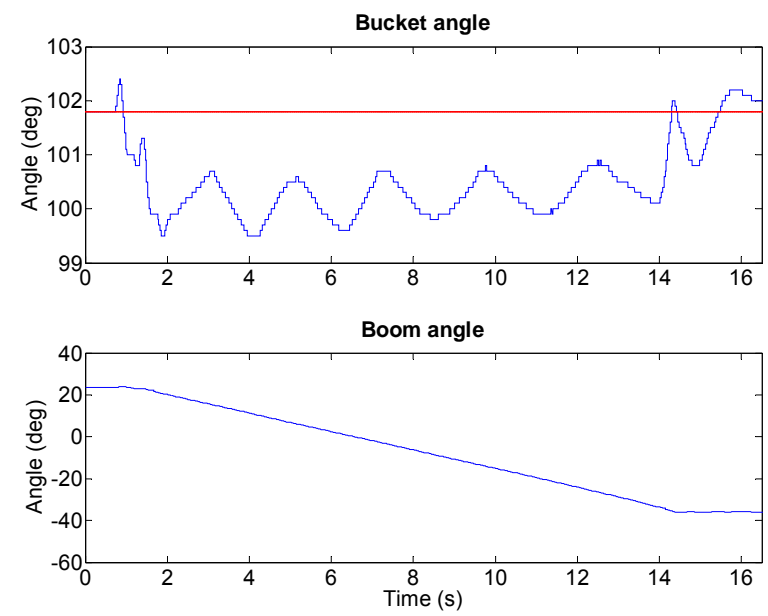

Fig. 8. Angles while the boom is moving downward with medium angular speed.

\section{Maximum Angular Speed}

There are no limitations used for valve opening by software when the maximum angular speed of the boom is measurement. The boom moved upward about 57 degrees in six seconds (Fig. 9). The tracking error is about five degrees and the steady-state error about 0.5 degrees. Accelerations of the boom are higher than in previous cases which can be noticed from higher peaks in the curve of the boom angle. On this account, also peaks in the angle of the bucket are higher.
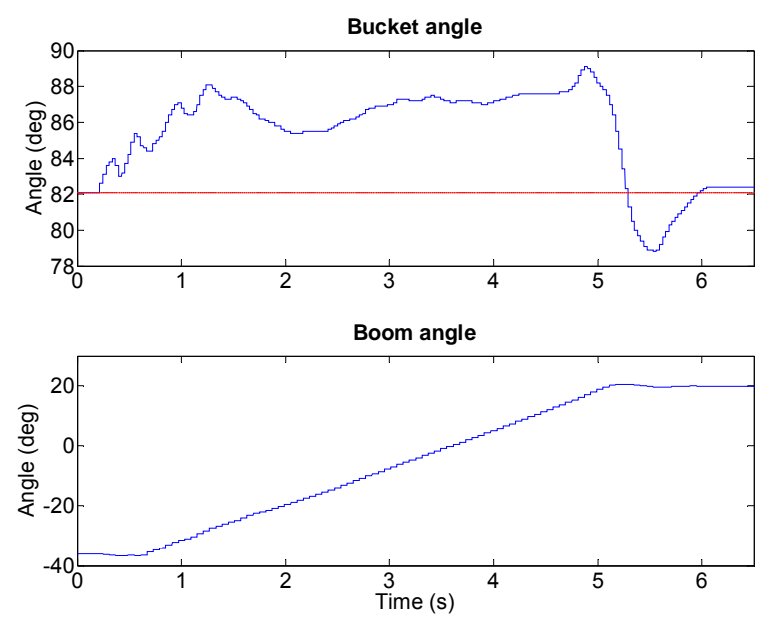

Fig. 9. Angles while the boom is moving upward without any limitations in the angular speed of the boom.

When the boom is moved downward without any limitations, the difference in the angular speed of the boom compared to previous cases is significant. The angle of the boom changes about 67 degrees in five seconds (Fig. 10). This is the worst situation since the machine is oscillating heavily. However, the maximum error is only about 6.5 degrees. If only the tracking error is considered, the error is about four degrees. The steady-state error is approximately zero.
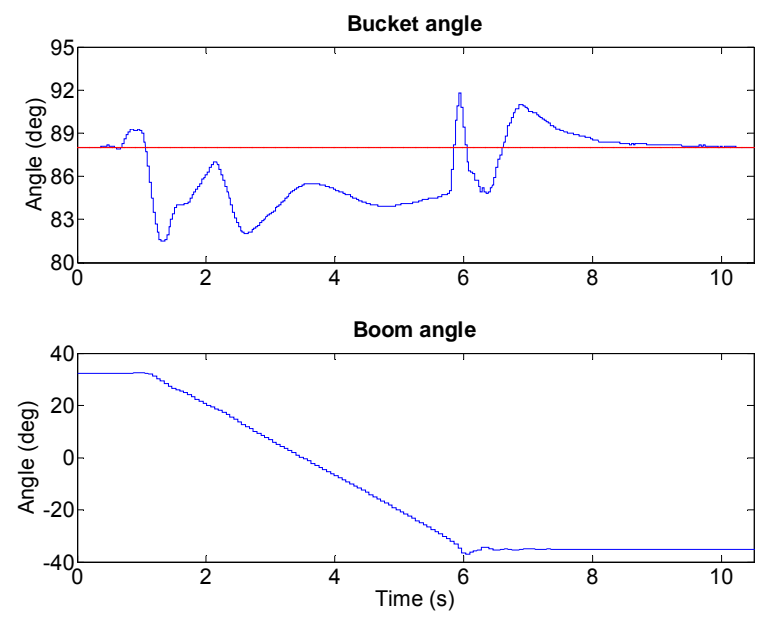

Fig. 10. Angles while the boom is moving downward without any limitations in the angular speed of the boom.

\section{Simulations}

Some oscillations can be noticed in the measurements. To find out if the reason for oscillations is in the measurement arrangement or in the controller some simulations were carried out. Simulations were made with verified simulation model of the proportional valve. The model has been developed in the Institute of Hydraulics and Automation. The model includes hydraulics and mechanics of the actuators. 

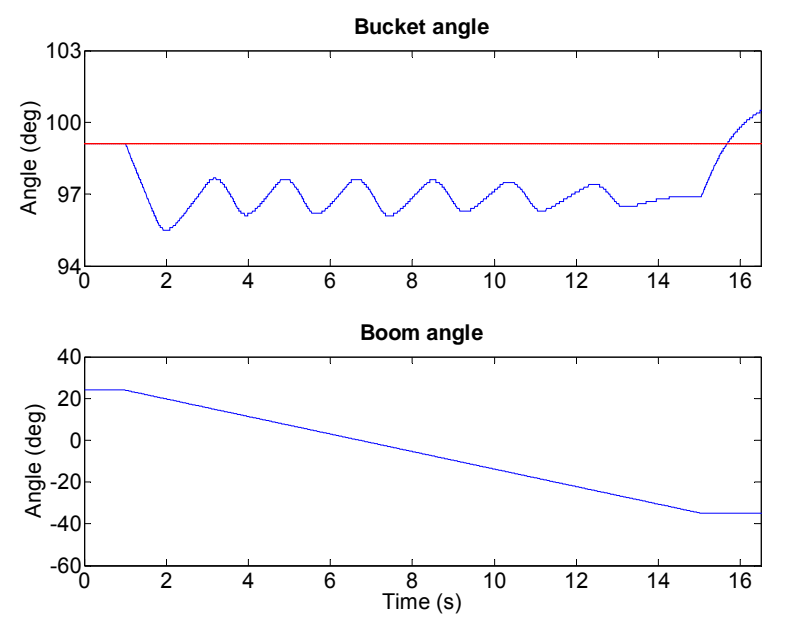

Fig. 11. Simulated change of the bucket angle when the boom is moving downward with medium speed. The control of the boom is similar in the simulation than it is in the measurements which are shown in Fig. 8.

Oscillations can be noticed easily when the boom is moving down with medium angular speed. Measured values of the bucket and the boom angles are plotted in Fig. 8. Simulated change of the bucket angle with similar control of the boom is plotted in the Fig. 11. It can be noticed by comparing Fig. 8 and Fig. 11 that shape (frequency and amplitude) of the simulated curve during the oscillation is similar to measured curve. There are no high-frequency peaks in the beginning and in the end of the simulated curve, since moving of the machine has not been taken into account in the simulation model. The high-frequency peaks are consequence of oscillation of the whole machine during high accelerations of the boom. This can be noticed from the curve of the boom angle. There are peaks in to the opposition direction during the starting and stopping the motion of the boom.

Similarity between measured and simulated values is so significant that it can be concluded that the oscillation is caused by either hydraulics or mechanics of the machine. It is probable that both factors have an influence.

\section{CONCLUSIONS}

Any changes to the hydraulics and mechanics of the studied machine have not been made to improve a behavior of this system. It was known already before testing the system that used proportional valve is not the best choice for this application. However, the stabilizer system was wanted to be a part of existing system. Electronics is distributed from original system. An accurate and robust inclinometer is used to measure the angle of the bucket. Electronics modules are communicating through CAN bus.

P-control is used since it is simple, stable and accurate enough for this application. Nonlinearities of the system, such as differential cylinder and dead zone of the valve are compensated by software.

Mechanical limitations of the machine have been taken care by measuring also the angle of the boom with inclinometer. If the angle of the bucket can not be compensated because of mechanical structure, the system stops the motion of the boom automatically and so prevents increasing error in the angle of the bucket.

The behavior of the system was measured to both directions, upward and downward with three different angular speed of the boom. Measurement results show that the error of the bucket angle is acceptable in all measurements. The highest peaks in curves are caused by oscillation of the whole machine during the acceleration of the boom. The controller can not filter these peaks. Only possible way to prevent these peaks is to limit accelerations of the boom. With the exception of these peaks the tracking errors and steady-state errors are small when the application is taken account.

The simulations support the theory that oscillation during actual control are caused by hydraulics and mechanics of the whole system. Specific reason for this small oscillation has to be found out in future.

Relatively slow and big valve and accuracy of the PWM signal are limiting the accuracy of the whole system. However, it can be stated that sufficient accuracy is achieved and the system is working properly.

\section{FUTURE WORK}

A reason for oscillations during the control has to be found out even though it is not disturbing the use of the system. By knowing the reason it may be possible to improve the behavior of the whole system. It is also important to find out the reason since it may influence to whole machine.

Ramp functions for boom control have to be improved. As it can be noticed from the measurements the whole machine is oscillating while high accelerations of the boom. The frequency of these high-frequency oscillations is so high that they can not be compensated with slow proportional valve. On this account, high accelerations have to be prevented.

The boom of the machine is telescope boom. By measuring the length of the boom and controlling the cylinder of the telescope, the bucket can be moved up and down so that horizontal distance between the bucket and front tires remains constant. By including this function into stabilizer system the bucket can be moved up and down in constant angle along a wall for instance. This can be useful function.

Also the user interface of the system has to be improved so that an operator does not have to disable and enable the system again when he or she wants to change the angle of the bucket. It could also be a useful function that the operator could give the value of the angle to where he wants to set the bucket and the system would control the bucket automatically into set value. The angle should also be shown on display.

The behavior of the system while driving the machine has to be tested. The valve is probably too slow to correct fast concussions but slow changes can be compensated. 


\section{ACKNOWLEDGMENT}

J. R. A. Uusisalo thanks Doctor M. P. Hyvönen from the Institute of Hydraulics and Automation for helping with simulations. Doctor M. P. Hyvönen has developed both the simulation model of the proportional valve and the model of the whole system. He also made simulations which helped to understand a behavior of the system.

\section{REFERENCES}

[1] Attachment range for studied mobile machine. Available: http://www.avanttecno.com/web/engtyoll/attachvalik.htm (24.5.2005)

[2] Valve specifications. Available: http://www.sauer-danfoss.com/ (20.4.2004)

[3] J. M. Vilenius, A. Raneda, M. P. Hyvönen, J. R. A. Uusisalo, and K. J. Huhtala, "Valve controlled teleoperated skid steered mobile machine," in Proc. 2004 ASME International Mechanical Engineering Congress and RD\&D Expo, Anaheim, 2004, IMECE2004-59257.

[4] Datasheet of the IO-modules. Available: http://www.axiomatic.com/i-omodule.pdf (31.5.2005)

[5] Datasheet of the DSP. Available:

http://www.freescale.com/files/dsp/doc/data_sheet/DSP56F803.pdf (30.5.2005)

[6] Datasheet of the inclinometer of the boom. Available: http://www.vti.fi/att/en/products/DN_SCA610_260804_pdf (30.5.2005)

[7] Datasheet of the inclinometer of the bucket. Available: http://www.axiomatic.com/inclinometer.pdf (30.5.2005) 